\title{
A Zernike-moment-based non-local denoising filter for cryo-EM images
}

\author{
WANG Jia \& YIN ChangCheng* \\ Department of Biophysics, School of Basic Medical Sciences, Peking University, Beijing 100191, China
}

Received October 31, 2012; accepted December 21, 2012

\begin{abstract}
Cryo-electron microscopy (cryo-EM) plays an important role in determining the structure of proteins, viruses, and even the whole cell. It can capture dynamic structural changes of large protein complexes, which other methods such as X-ray crystallography and nuclear magnetic resonance analysis find difficult. The signal-to-noise ratio of cryo-EM images is low and the contrast is very weak, and therefore, the images are very noisy and require filtering. In this paper, a filtering method based on non-local means and Zernike moments is proposed. The method takes into account the rotational symmetry of some biological molecules to enhance the signal-to-noise ratio of cryo-EM images. The method may be useful in cryo-EM image processing such as the automatic selection of particles, orientation determination, and the building of initial models.
\end{abstract}

cryo-electron microscopy, non-local means, Zernike moments, rotational symmetry

Citation: Wang J, Yin C C. A Zernike-moment-based non-local denoising filter for cryo-EM images. Sci China Life Sci, 2013, 56: 384-390, doi: $10.1007 / \mathrm{s} 11427-013-4467-3$

In recent decades, cryo-electron microscopy (cryo-EM) has become an important means of studying the structure of biological macromolecules, viruses, and even the whole cell. Cryo-EM can keep biological molecules in their native states. However, cryo-EM images must be obtained under low-dose conditions to avoid radiation damage to the sample. Biological samples are composed of light atoms, and cryo-EM images thus have very low contrast and signal-to-noise (SNR) ratios. To analyze the images and obtain the structure of a sample, image processing must be performed. For example, it is easier to select particles after enhancing the image contrast by filtering the original images. Several image filtering methods used to denoise cryo-EM images have been proposed, such as the non-linear anisotropic diffusion filter [1], the bilateral filter [2], and the optimized locally adaptive non-local means filter (LANL) [3].

*Corresponding author (email: ccyin@ hsc.pku.edu.cn)
The non-local means filter [4] is a new kind of spatial filter that works well in both detail preservation and noise removal, and it is capable of yielding high-quality denoising results. Improvements of this method have been reported to work well in the processing of different kinds of images, such as fluorescent images of living cells [5] and Positron Emission Tomography images [6]. The improvements include parameter selection based on the characteristics of different samples and a decrease in the computational cost. A speed-up approach introduced by Liu et al. [7] integrates both mirror images and Laplacian pyramids and is 50 times faster than the classic non-local means algorithm. The LANL method based on the non-local means filter works well in smoothing the background. Some biological molecules have rotational symmetry but the original LANL method did not consider this situation. If the rotation symmetry is considered, the LANL method may be improved. This can be achieved using Zernike moments to compute the similarity between two pixels in an image. Zernike mo- 
ments have the property of rotation invariance; i.e., the values of Zernike moments do not change after rotating the given image through any angle.

In this paper, we present a Zernike-moment-based non-local means filter for cryo-EM images and analyze the filtering effect. In the next two sections, we briefly introduce the non-local means filter and Zernike moments. Section 3 describes our methods. Section 4 gives the results, which include examinations of our method and analyses on how the parameters affect the filtering effect, as judged by the peak SNR (PSNR) and masked peak SNR (MPSNR). Section 5 concludes the paper and discusses proposals for future work.

\section{Non-local means filter}

Digital image filters [8] can be generally divided into spatial-domain filters and frequency-domain filters, and most of the former filters are based on local areas of the image. In recent years, a new spatial-domain filter based on non-local areas of the image was proposed by Buades [9]. The basic principle of the non-local means filter is that for a certain pixel $i$ and any other pixel $j$ in the image, if $i$ and $j$ have similar neighboring pixels then pixel $j$ has a larger weight in determining the value $i$ in filtering. The pixel $i$ and its neighborhood are called the comparison window. It is time consuming to compute all the pixels in the image, and we therefore only consider a certain area around pixel $i$. This area is called the search window. For a noisy image $I,\{v(i)$, $i \in I\}$ denotes all its pixel values; after filtration, the corresponding pixel value is $u(i)$ :

$$
u(i)=\sum_{j \in I} \omega(i, j) v(j)
$$

$\{\omega(i, j)\}$ is the weight of all other pixels in image $I$, denoting the similarity of pixels $i$ and $j, \omega$ ranges $0 \leqslant \omega \leqslant 1$ and $\Sigma_{j} \omega(i, j)=1$. The weight $\omega(i, j)$ is based on the similarity of $v\left(N_{i}\right)$ and $v\left(N_{j}\right)$, which denote the two neighborhoods of pixels $i$ and $j$ :

$$
\begin{gathered}
\omega(i, j)=\frac{1}{Z(i)} \exp \left(-\frac{\left\|v\left(N_{i}\right)-v\left(N_{j}\right)\right\|_{2, \alpha}^{2}}{h^{2}}\right) \\
E(i, j)=\left\|v\left(N_{i}\right)-v\left(N_{j}\right)\right\|_{2, \alpha}^{2}=\sum_{\delta \in N} G_{\alpha}(\delta)[v(i+\delta)-v(j+\delta)]^{2} \\
Z(i)=\sum_{j} \exp \left(-\frac{\left\|v\left(N_{i}\right)-v\left(N_{j}\right)\right\|_{2, \alpha}^{2}}{h^{2}}\right)
\end{gathered}
$$

$E(i, j)$ is the Gaussian weighted Euclidean distance and $Z(i)$ is the normalization factor; therefore, $\Sigma_{j} \omega(i, j)=1$. Pa- rameter $h$ controls the decay of the exponential function, and thus adjusts the filtering effect, which depends on the image to be filtered. The definition of $h$ in the LANL method is $h=\beta \sigma$, where $\beta$ is a constant and $\sigma$ is the standard deviation of the image with a threshold [3]. The LANL method has a threshold for parameter $h$, so that it cannot be too large or too small.

\section{Zernike moments}

The moment technique is widely used in the field of image processing [10,11], such as image reconstruction, image compression, edge detection, and object identification. Zernike moments are series of orthogonal basis functions based on Zernike polynomials [12-14], which have the property of rotation invariance. Zernike moments can be computed to arbitrarily high order, and moments of different orders correspond to independent characteristics of the image. Generally speaking, higher order moments give more detailed shape characteristics of the image. Mathematically, Zernike basis functions are defined with an order $p$ and a repetition $q$ over $D=(p, q)|0 \leqslant p \leqslant \infty| q,|\leqslant p, p-| q \mid=$ even.

$$
\begin{gathered}
Z_{p q}=\frac{p+1}{\pi} \iint_{x^{2}+y^{2}=1} V_{p q}^{*}(x, y) f(x, y) \mathrm{d} x \mathrm{~d} y, \\
V_{p q}^{*}=\mathrm{e}^{-i q \theta} \sum_{k=|q||| p-k \mid=\operatorname{even}}^{p} \frac{(-1)^{\left(\frac{p-k}{2}\right)\left(\frac{p+k}{2}\right) !}}{\left(\frac{p-k}{2}\right) !\left(\frac{k-q}{2}\right) !\left(\frac{k+q}{2}\right) !} \rho^{k},
\end{gathered}
$$

where $\rho$ is the length of the vector from the origin to pixel $(x, y)$, and $\theta$ is the angle between vector $\rho$ and the $x$-axis in the counterclockwise direction. For a digital image $I$, the Zernike moments become

$$
Z_{p q}=\frac{p+1}{\pi} \sum_{x} \sum_{y} V_{p q}^{*}(x, y) I(x, y)
$$

with the condition $x^{2}+y^{2} \leqslant 1$.

\section{Methods}

Considering that some biological molecules have rotational symmetry, the original non-local means algorithm does not work well in filtering such images. We therefore use Zernike moments to compute the similarity of two comparison windows. That is, we use Zernike moments instead of the Gaussian weighted Euclidean distance to compute the distance between two pixels. Zernike moments are complex moments and so we use the moduli of Zernike moments. The moduli of Zernike moments of the original image and the rotated image have the same value. In our method, the 
weight for the relation between two pixels becomes

$$
\begin{gathered}
\omega(i, j)=\frac{1}{Z(i)} \exp \left(-\frac{\left|\operatorname{Zer}\left(N_{i}\right)\right|-\mid \operatorname{Zer}\left(N_{j}\right) \|^{2}}{h^{2}}\right), \\
Z(i)=\sum_{j} \exp \left(-\frac{\left\|\operatorname{Zer}\left(N_{i}\right)|-| \operatorname{Zer}\left(N_{j}\right)\right\|^{2}}{h^{2}}\right) .
\end{gathered}
$$

$\left|\operatorname{Zer}\left(N_{i}\right)\right|$ and $\left|\operatorname{Zer}\left(N_{j}\right)\right|$ denote the moduli of Zernike moments of the two neighborhoods of pixels $i$ and $j$, and $Z(i, j)$ is the normalization factor.

The comparison window $(c w)$, search window $(s w)$ and orders of Zernike moments $(p, q)$ should be different because of the low SNR of cryo-EM images and the different shapes of different biological samples. In our method, we do not specify the value of parameter $q$, so the value of $q$ is taken over all possible values depending on parameter $p$. For example, when $p$ takes the value $2, q$ takes the value 0 and 2. In this paper, we also analyze how these different parameters affect the filtering effect.

Another parameter is the filtering degree factor $h$. If the parameter $h$ is small, almost all other weights are close to zero and the weight of the pixel itself is close to one, and there is no filtering at all. Buades et al. [9] suggested that $h$ should be selected between $(10 \sigma)^{1 / 2}$ and $(15 \sigma)^{1 / 2}$, where $\sigma$ is the standard deviation of the additive Gaussian noise contained in the image. Here we use $(12 \sigma)^{1 / 2}$. Additionally, the thickness of ice varies in the sample, which affects the value of $\sigma$ within a cryo-EM image [3]; hence, we also propose a locally adaptive variable factor depending on the local standard deviation. In our method, the parameter $h$ is defined as

$$
h=\left\{\begin{array}{l}
0.5 \sqrt{12 \sigma_{I}}, \sigma_{N} \leqslant 0.5 \sigma_{I}, \\
1.5 \sqrt{12 \sigma_{I}}, \sigma_{N} \geqslant 1.5 \sigma_{I}, \\
\sqrt{12 \sigma_{N}}, \text { others, }
\end{array}\right.
$$

where $\sigma_{N}$ and $\sigma_{I}$ are the standard deviations for the neigh- borhood and the whole image, respectively.

Figure 1 shows the difference in weights between our method and the non-local means method. A, B and C are three comparison windows when determining the weights and the center of $\mathrm{C}$ is the target pixel. $\mathrm{B}$ and $\mathrm{C}$ represent the same subunits in biological molecules. When determining the weights of $A$ and $B$, our method assigns a higher weight to $\mathrm{B}$ than to A. Therefore, our method considers that the pixel in the center of B is similar to the target pixel.

To test our method, we chose a reconstruction density map of GroEL with resolution $6 \AA$ (EMDB, accession code 1081) to generate simulation data. We then used a crosssection of the three-dimensional (3D) density map and added random Gaussian noise with an SNR of 0.1 , which is close to the SNR of a real cryo-EM image. The SNR is defined as

$$
\mathrm{SNR}=\frac{\operatorname{var}(u)}{\operatorname{var}(n)},
$$

where $\operatorname{var}(u)$ and $\operatorname{var}(n)$ denote the standard deviations of $u$ and $n$, respectively. We filtered the noisy image with our method and the LANL-means filter and then computed the PSNR and MPSNR between the original noise-free image and the filtered image. For an 8-bit grayscale image, the PSNR is defined as

$$
\mathrm{PSNR}=10 \lg \frac{255^{2}}{\mathrm{MSE}}
$$

where MSE is the root-mean-square error calculated by comparing the original noise-free image and the denoised image. To obtain the MPSNR, a circle with radius slightly larger than the radius of GroEL as a mask was used to exclude the non-signal part.

Next we filtered real cryo-EM data using our method, and then reconstructed the $3 \mathrm{D}$ structure using the filtered data. The original data were downloaded from http://blake. grid.bcm.edu/dl/EMAN11/eman2_2011_demo_data.tgz and we used GroEL having D7 symmetry. All the filter scripts of our method were implemented in MATLAB [15], which has many built-in image functions. We used parallel
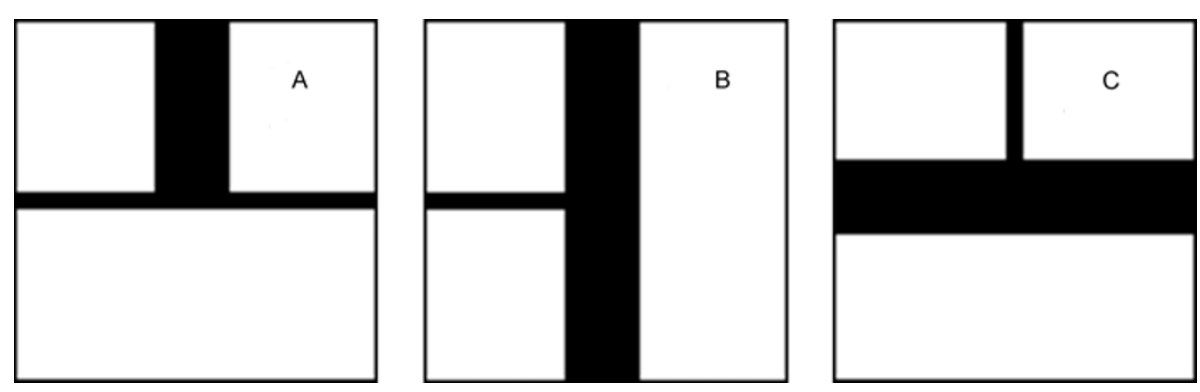

Figure 1 The centers of A, B and C are different pixels. B and C have similar pixel neighborhoods, and can represent the same subunits in biological molecules, but the original non-local means method gives a lower weight than our method; A and $\mathrm{C}$ are different pixel neighborhoods, and the non-local means method gives a higher weight than our method. 
MATLAB programming to accelerate our filtering method [16]. We then reconstructed the 3D structure with the application of EMAN2 [17] using default parameters. The protocols were downloaded from http://blake.bcm.edu/ emanwiki/Ws2011/Eman2.

\section{Results}

\subsection{Simulation data}

We first studied the difference in applying the optimized LANL means filter and our method to simulation data. The 60th section (parallel to the $x y$-plane) along the $z$-axis of the original 3D reconstruction density map of GroEL [18] (EMDB, accession code 1081) was chosen as the signal (Figure 2A). We then added Gaussian random noise with a SNR of 0.1, which is similar to the SNR of real data, to generate the simulation data (Figure 2B). Figure 2 shows the filtering results for optimized LANL means filter and our method. Although the background obtained with the optimized LANL means filter (Figure 2C and E) was smoother than that obtained with our method (Figure 2D and F), the signal part of the image was smooth in both cases. When determining the value of a certain pixel after filtering, our method obtains more similar pixels than the LANL filter in the range of the search window, especially at the curved edge of the signal, owing to the property of rotation invariance of Zernike moments. The center black spot of the images filtered using our method, for example, is clearer than that obtained with the LANL filter.

To quantitatively distinguish the two methods, we com- pared the PSNR and MPSNR. Figure 3 compares the PSNR and MPSNR between the optimized LANL means filter and our method. It shows the influence of the parameter search window and comparison window on the PSNR and MPSNR. The horizontal axis denotes the size of the search window and comparison window. The first number is the size of the search window and the second is the size of the comparison window. For example, 5-3 denotes a search window $s w=5$ and a comparison window $c w=3$. The vertical axis is the SNR $(\mathrm{db})$. The values of parameter $\mathrm{p}$ in our method are 0,2 and 4. psnr-zer and mpsnr-zer are the values of PSNR and MPSNR using our method and psnr-lanl and mpsnr-lanl are corresponding values using the LANL method. Although the PSNR of our method is lower than that of the optimized LANL means filter at most of the given points, the value of MPSNR of our method is higher in the range investigated. This means that our method can keep more signals than the optimized LANL means filter.

\subsection{Real data}

We used real cryo-EM data, a total of 4234 GroEL particle images, to test our method. We randomly selected particles from the data set to form four groups and the number of particles in each group was different. We used the four groups of data to reconstruct the 3D structure using EMAN2 [18]. In this procedure, we used the same parameters and data sets in that protocols and we obtained four different reconstructions. The four maps were refined using default phase-flipped hp data sets. Next we filtered the images in the four groups using our method and the parameters $\mathrm{cm}=15$,

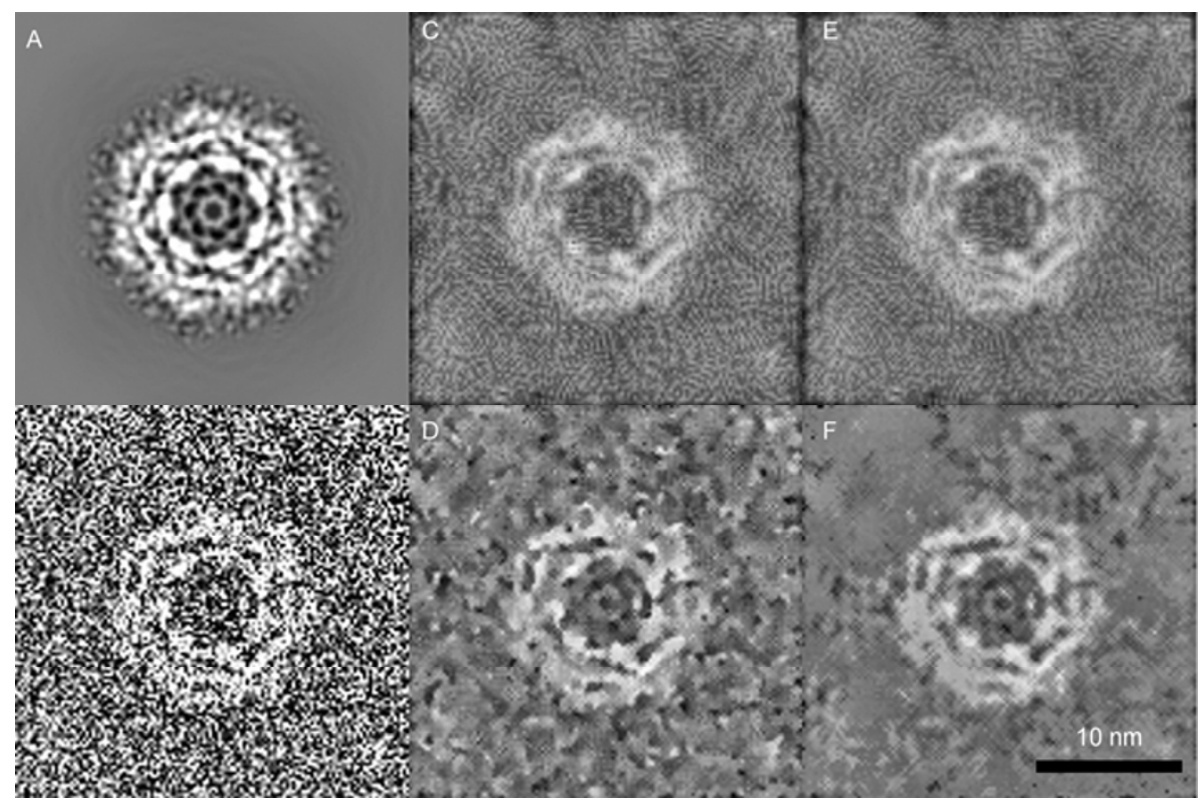

Figure 2 Montages compare results obtained with the LANL filter and our method. A, Original image, the 60th section along the $z$-axis of the 3D density map of GroEL. B, Image A corrupted by Gaussian random noise at SNR=0.1. C, Image B filtered by an LANL filter with $d=3, s=5, \beta=4$. D, Image B filtered using our method with $c w=3, s w=5, p=2, q=0,2$. E, Image B filtered with an LANL filter with $d=5, s=15, \beta=4$. F, Image B filtered using our method with $c w=5, s w=15, p=2, q=0,2$. 


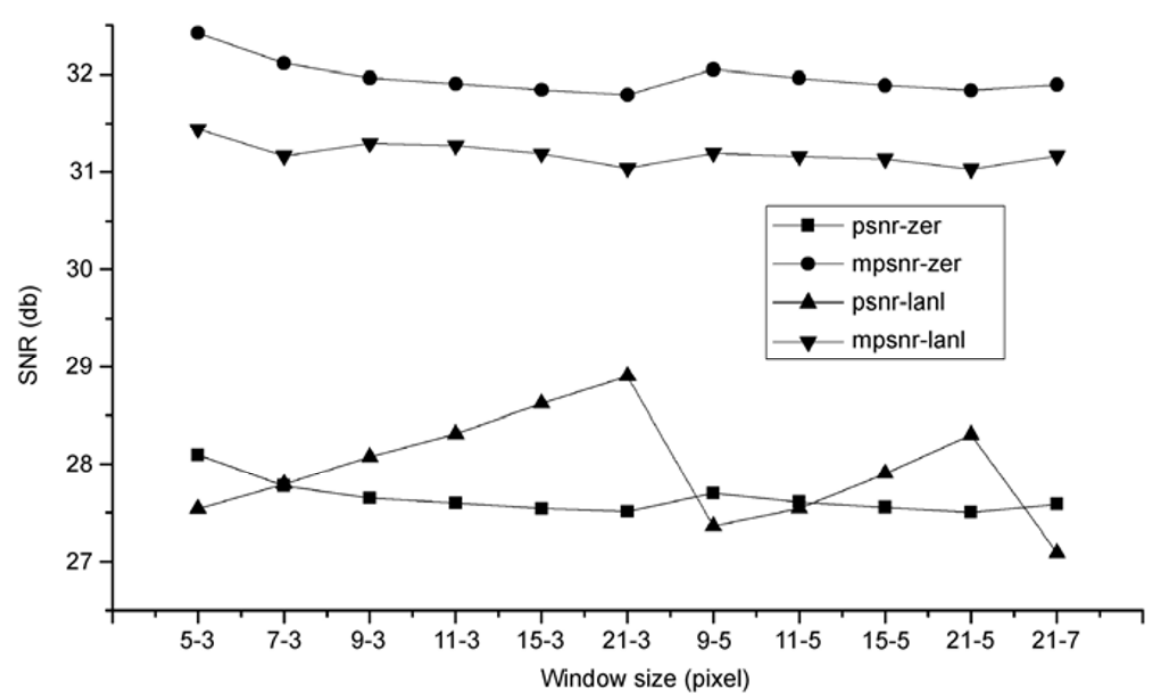

Figure 3 Influence of the search window/comparison window on the PSNR and MPSNR. The first number is the size of the search window and the second is the size of the comparison window. For example, 5-3 denotes a search window $s w=5$ and comparison window $c w=3$. The values of parameter $\mathrm{p}$ in our method are 0,2 and 4 .

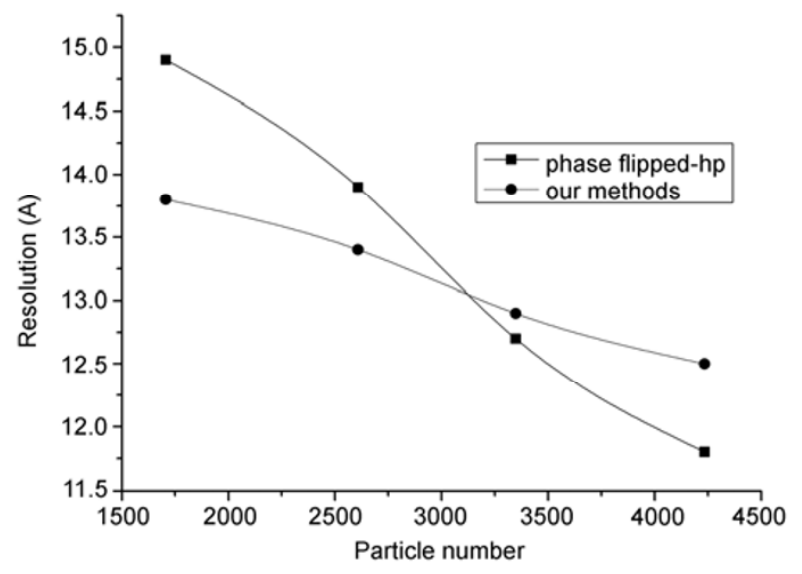

Figure 4 Particle number dependence of the 3D reconstruction resolution. It is concluded that our method achieves higher resolution when the particle number is small.

$s w=5$ and $p=4(q=0,1,2)$. We then used the filtered data to reconstruct $3 \mathrm{D}$ structure (starting from the "make initial model" step). Figure 4 shows the resolution ( $\mathrm{FSC}=0.5$ ) after running e2eotest in EMAN2. Figure 5 shows the surfaces of some of the reconstruction maps and Figure 6 shows cross-sections of the reconstruction maps. It is seen that our method achieved higher resolution when the particle number was small. The density map of B, which is reconstructed from 1706 particle images filtered using our new method, for example, looks like density map $\mathrm{C}$, which is reconstructed from 4234 particle images of the phase- flipped hp data set.

To test our method in terms of maintaining a signal, we calculated cross-correlation coefficient (CCC) [19,20] for correlation between different reconstructions (Table 1). This image processing was carried out with SPIDER [21]. The first column gives the values of the CCC for different density maps reconstructed with different particle numbers and a density map with resolution of $8.4 \AA$, which was obtained from the high-resolution refinement in that protocol. The second column gives values of the CCC for reconstructions from images filtered using our method and the 8.4- $\AA$ density map. The third column gives values of the CCC for reconstructions from phase-flipped hp images and images filtered using our method. It is evident that our filtering method performs well in keeping the signal (Figures 5 and 6, Table 1).

\section{Discussion and conclusion}

In this paper, we proposed a Zernike-moment-based nonlocal means filter method for application to low-SNR cryo-EM images. We use Zernike moments, which have the feature of rotation invariance, to weigh the distance between two pixels. This method can improve the SNR without sacrificing signal details in the original images. It is concluded that the sizes of the search window and comparison window affect the smoothness of the background. Generally speaking, a larger search window provides a smoother background (Figure 2D and F). This might be because of the characteristics of the non-local means filter. When the search window is bigger, more pixels are considered when determining the value of the target pixel.

The orders of Zernike moments also greatly affect the filter results. A higher order of Zernike moments will give more detail in the image. In this paper, we used a lower order to improve the computation speed and obtain a smoother background.

There are problems in applying our method in image 

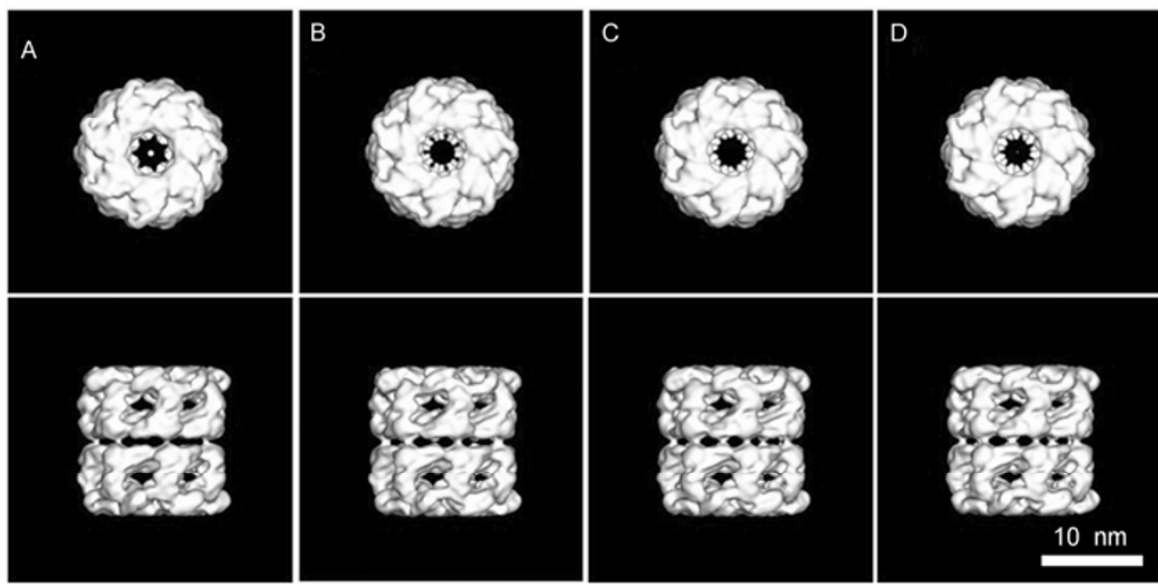

Figure 5 Top and side views of the reconstructed 3D density maps. All the images are displayed using e2display.py with the same threshold. A, 3D density map reconstructed from the phase-flipped hp group 1 data sets (particle number of 1706). B, 3D density map reconstructed from the same data sets filtered using our method. C, 3D density map reconstructed from the phase-flipped hp group 4 data sets (particle number of 4234). D, 3D density map reconstructed from the same data sets filtered using our method.
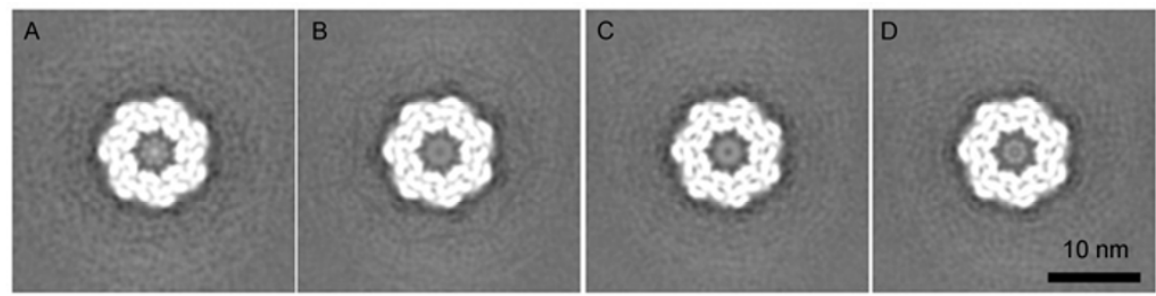

Figure 6 The 79th section (parallel to the $x y$-plane) along the $z$-axis of the density maps. A, 3D density map reconstructed from the phase-flipped hp group 1 data sets (particle number of 1706). B, 3D density map reconstructed from the same data sets filtered using our method. C, 3D density map reconstructed from the phase-flipped hp group 4 data sets (particle number of 4234). D, 3D density map reconstructed from the same data sets filtered using our method.

Table 1 Cross-correlation coefficient (CCC) for correlation between different reconstructions

\begin{tabular}{ccccc}
\hline Group & Particle number & $\mathrm{CCC}^{\mathrm{a})}$ & $\mathrm{CCC}^{\mathrm{b})}$ & $\mathrm{CCC}^{\mathrm{c})}$ \\
\hline 1 & 1706 & 0.72912 & 0.73843 & 0.94960 \\
2 & 2608 & 0.74071 & 0.76462 & 0.95837 \\
3 & 3348 & 0.77526 & 0.77025 & 0.97114 \\
4 & 4234 & 0.78628 & 0.77537 & 0.96521 \\
\hline
\end{tabular}

a) Values of CCC for the density maps reconstructed from data sets filtered using phase-flipped hp data and a density map with resolution of $8.4 \AA$, which was obtained from the high-resolution refinement in that protocol. b) Values of CCC for the density maps reconstructed from data sets filtered using our method and the 8.4- $\AA$ density map. c) Values of CCC for the density maps reconstructed from data sets filtered using phase-flipped hp data and our method.

processing. One of these problems is the determination of the values of parameters, and especially the order of Zernike moments, which can greatly affect the PSNR of the images. Generally speaking, lower orders of Zernike moments are suitable for cryo-EM images. Another problem is that the non-local filter is more time-consuming than other filters. It takes about 15 seconds to compute an image of $512 \times 512$ pixels on a personal computer. Here we used parallel programming [22] in MATLAB. This problem may be overcome by using a graphics processing unit GPU [23] or employing another acceleration method.

In conclusion, the Zernike-moment-based non-local means filter considers both the rotation invariance of some biological molecules and the influence of the thickness of ice in the cryo-EM image. This method works well in suppressing noise and enhancing signal details. The filtering method is most effective when the particle number is low (Figure 4, Table 1) compared with other filtering methods. The method may be useful in selecting particle images, determining the orientation of particles and deducing initial models of biological complexes from cryo-EM images.

This work was supported by the National Basic Research Program of China (2010CB912400). 
1 Fernandez J J, Li S. An improved algorithm for anisotropic nonlinear diffusion for denoising cryo-tomograms. J Struct Biol, 2003, 144: $152-161$

2 Jiang W, Baker M L, Wu Q, et al. Applications of a bilateral denoising filter in biological electron microscopy. J Struct Biol, 2003, 144: 114-122

3 Wei D Y, Yin C C. An optimized locally adaptive non-local means denoising filter for cryo-electron microscopy data. J Struct Biol, 2010 , 172: 211-218

4 Buades A, Coll B, Morel J M. A non-local algorithm for image denoising. In: IEEE Computer Society Conference on Computer Vision and Pattern Recognition, 2005. 2: 60-65

5 Yang L, Parton R, Ball G, et al. An adaptive non-local means filter for denoising live-cell images and improving particle detection. J Struct Biol, 2010, 172: 233-243

6 Yin Y, Lu J. A non-local means approach for PET image denoising. In: World Congress on Medical Physics and Biomedical Engineering, 2010. 25: 127

7 Liu Y L, Wang J, Chen X, et al. A robust and fast non-local means algorithm for image denoising. J Comput Sci Technol, 2008, 23: 270-279

8 Gonzalez R C, Woods R E. Digital Image Processing. 2nd ed. Upper Saddle River: Prentice Hall, 2002. 59-169

9 Buades A, Coll B, Morel J M. A review of image denoising algorithms, with a new one. Multiscale Model Sim, 2006, 4: 490-530

10 Mandal M K, Aboulnasr T, Panchanathan S. Image indexing using moments and wavelets. IEEE Trans Consum Electron, 1996, 42: $557-565$

11 Khotanzad A, Hong Y H. Invariant image recognition by Zernike moments. IEEE Trans Patt Anal Mach Intell, 1990, 12: 489-497

12 Prokop R J, Reeves A P. A survey of moment-based techniques for unoccluded object representation and recognition. CVGIP: Graph
Models Image Pro, 1992, 54: 438-460

13 Ji Z, Chen Q, Sun Q S, et al. A moment-based nonlocal-means algorithm for image denoising. Inf Proc Lett, 2009, 109: 1238-1244

14 Grewenig S, Zimmer S, Weickert J. Rotationally invariant similarity measures for nonlocal image denoising. J Vis Commun Image Represent, 2011, 22: 117-130

15 Gonzalez R C, Woods R E, Eddins S L. Digital Image Processing Using MATLAB. Beijing: Publishing House of Electronics Industry, 2004. 5-21

16 Choy R, Edelman A. Parallel MATLAB: doing it right. Proc IEEE, 2005, 93: 331-341

17 Tang G, Peng L, Baldwin P R, et al. EMAN2: an extensible image processing suite for electron microscopy. J Struct Biol, 2007, 157: 38-46

18 Ludtke S J, Chen D H, Song J L, et al. Seeing GroEL at 6 A resolution by single particle electron cryomicroscopy. Structure, 2004, 12 : 1129-1136

19 Frank J, Al-Ali L. Signal-to-noise ratio of electron micrographs obtained by cross correlation. Nature, 1975, 256: 376-379

20 Frank J. Three-dimensional electron microscopy of macromolecular assemblies: visualization of biological molecules in their native state. New York: Oxford University Press, 2006. 94-100

21 Frank J, RadermacherPawel M, Zhu J, et al. SPIDER and WEB: processing and visualization of images in 3D electron microscopy and related fields. J Struct Biol, 1996, 116: 190-199

22 Gao W, Kemao Q, Wang H, et al. Parallel computing for fringe pattern processing: a multicore CPU approach in MATLAB $<$ sup $>\AA</$ sup $>$ environment. Opt Laser Eng, 2009, 47: 1286-1292

23 Fan Z, Qiu F, Kaufman A, et al. GPU cluster for high performance computing. In: Proceedings of the ACM/IEEE Supercomputing Conference, Pittsburgh, USA, 2004. 47-47

Open Access This article is distributed under the terms of the Creative Commons Attribution License which permits any use, distribution, and reproduction in any medium, provided the original author(s) and source are credited. 